QUARTERLY OF APPLIED MATHEMATICS

VOLUME LXIX, NUMBER 3

SEPTEMBER 2011, PAGES 405-424

S 0033-569X(2011)01211-1

Article electronically published on April 4, 2011

\title{
MULTIPLE SOLUTIONS FOR HYDROMAGNETIC FLOW OF A SECOND GRADE FLUID OVER A STRETCHING OR SHRINKING SHEET
}

\author{
BY \\ ROBERT A. VAN GORDER (Department of Mathematics, University of Central Florida, Orlando, \\ Florida 32816) \\ AND \\ K. VAJRAVELU (Department of Mathematics, University of Central Florida, Orlando, Florida \\ 32816)
}

\begin{abstract}
We study a class of fourth-order nonlinear differential equations arising in the hydromagnetic flow of a second grade fluid over a stretching or shrinking sheet. Explicit exact solutions are obtained. Furthermore we show that the differential equation may admit zero or one or two physically meaningful solutions depending on the values of the physical parameters of the model. As a special case, we recover the single or the dual solutions and compare them with the available results in the literature. Also, the obtained multiple solutions for several sets of values of the parameters are presented through tables and graphs, and the qualitative behaviors are discussed.
\end{abstract}

1. Introduction. During the past three decades there have been several studies of boundary layer flows of non-Newtonian fluids. These investigations have been for nonNewtonian fluids of the differential type (see [1]). In the case of fluids of differential type, the equations of motion are an order higher than the Navier-Stokes equations, and thus the adherence boundary condition is insufficient to determine the solution completely (see [2]-4] for a detailed discussion of the relevant issues). The same is also true for the approximate boundary layer approximations of the equations of motion. In the absence of a clear means of obtaining additional boundary conditions, Beard and Walters [5], in their study of an incompressible fluid of second grade, suggested a method for overcoming this difficulty. They suggested a perturbation approach in which the velocity and the pressure fields were expanded in a series in terms of a small parameter $\epsilon$. Though this

Received August 15, 2009.

2000 Mathematics Subject Classification. Primary 76D03.

Key words and phrases. Similarity solution, stretching sheet, shrinking sheet, Navier-Stokes equations, exact solution, hydromagnetic flow, viscoelastic fluid, second grade fluid, multiple solutions.

E-mail address: rav@knights.ucf.edu

E-mail address: vajravel@pegasus.cc.ucf.edu

(C)2011 Brown University Reverts to public domain 28 years from publication 
approximation reduces the order of the equation, it treats a singular perturbation problem as a regular perturbation problem.

In 1991, Garg and Rajagopal [6] suggested that it would be preferable to overcome the difficulty associated with the paucity of boundary conditions by augmenting them on the basis of physically reasonable assumptions. They thought that it is possible to do this in the case of flows which take place in unbounded domains by using the fact that either the solution is bounded or the solution has a certain smoothness at infinity. To demonstrate this, Garg and Rajagopal [6 studied the stagnation flow of a fluid of second grade by augmenting the boundary conditions. Their results agreed well with the results of Rajeswari and Rathna [7] who studied the problem based on the perturbation approach, for a small value of the perturbation parameter. The advantage of augmenting the boundary conditions over the perturbation approach is that the analysis is valid even for large values of the parameter $\epsilon$, and significant deviations from the Newtonian behavior are possible for even moderately large values of $\epsilon$.

The Cauchy stress $\mathbf{T}$ in an incompressible homogeneous fluid of second grade has the form (see [8])

$$
\mathbf{T}=-p \mathbf{I}+\mu \mathbf{A}_{1}+\alpha_{1} \mathbf{A}_{2}+\alpha_{2} \mathbf{A}_{1}^{2}
$$

where

$$
\mathbf{A}_{1}=(\operatorname{grad} \mathbf{v})+(\operatorname{grad} \mathbf{v})^{T}
$$

and

$$
\mathbf{A}_{2}=\frac{\mathrm{d} \mathbf{A}_{1}}{\mathrm{~d} t}+\mathbf{A}_{1}(\operatorname{grad} \mathbf{v})^{T} \mathbf{A}_{1}
$$

In the above equations, the spherical stress $-p \mathbf{I}$ is due to the constraint of incompressibility, $\mu$ is the viscosity, $\alpha_{1}$ and $\alpha_{2}$ are material moduli and usually referred to as the normal stress moduli, $\mathrm{d} / \mathrm{d} t$ denotes the material time derivative, $\mathbf{v}$ denotes the velocity field, and $\mathbf{A}_{1}$ and $\mathbf{A}_{2}$ are the first two Rivlin-Ericksen tensors. The above model has been studied in great detail. The sign of the coefficient has been the subject of much controversy, and a thorough discussion of the issues involved can be found in the recent critical review of Dunn and Rajagopal [9]. We shall not get into a discussion of these issues here. In this study we shall assume that Eq. (1) models the fluid exactly. If the fluid modeled by Eq. (1) is to be compatible with thermodynamics, in the sense that all motions of the fluid meet the Clausius-Duhem inequality and the assumption that the specific Helmholtz free energy of the fluid is a minimum when the fluid is locally at rest, then

$$
\mu \geq 0, \alpha_{1} \geq 0 \text {, and } \alpha_{1}+\alpha_{2}=0 \text {. }
$$

In 1984, Rajagopal et al. [10] studied the Falkner-Skan flows of a non-Newtonian fluid. Later, Vajravelu and Rollins [11] studied the flow and heat transfer in an incompressible second-order fluid due to stretching of a plane elastic surface. Vajravelu and Rollins examined the effects of viscous dissipation and internal heat generation or absorption in a viscoelastic boundary layer flow. Recently, Sarma and Rao [12] analyzed the effects of work due to deformation in the energy equation. In [11, 12] (also see [13]-[16]), the sign for the material constant $\alpha_{1}$ (in Eq. (3)) was taken as negative; however, this is not compatible with the stability criteria (see 9]). Very recently, Vajravelu and Roper [17. studied the flow and heat transfer in a viscoelastic fluid over a stretching sheet 
with power law surface temperature, including the effects of viscous dissipation, internal heat generation or absorption, and work due to deformation in the energy equation. Furthermore, they augmented the boundary conditions and used the proper sign for the material constant $\left(\alpha_{1} \geq 0\right)$, and analyzed the salient features of the flow and heat transfer characteristics. Then, Vajravelu and Rollins [18 extended a specialized case of Vajravelu and Roper [17] to an electrically conducting fluid permeated by a uniform transverse magnetic field with uniform suction at the wall. This problem has definite applications to polymer technology (where one deals with stretching plastic sheets) and metallurgy where hydromagnetic techniques have been recently used. To be more specific, it may be pointed out that many metallurgical processes involve the cooling of continuous strips or filaments by drawing them through a quiescent fluid and that in the process of drawing, these strips are sometimes stretched. Mention may be made of drawing, annealing and tinning of copper wires. In all these cases the properties of the final product depend to a great extent on the rate of cooling. By drawing such strips in an electrically conducting fluid subject to a magnetic field, the rate of cooling can be controlled and final products of desired characteristics might be achieved. Another interesting application of hydromagnetics to metallurgy lies in the purification of molten metals from nonmetallic inclusions by the application of a magnetic field (see [19]).

In the present paper, we consider a more general boundary value problem than that considered by Vajravelu and Rollins [18. In particular, we allow for suction or injection at the stretching surface. We find that, in instances of injection, there may be multiple physical solutions for the stretching sheet problem. Furthermore, we allow for the stretching parameter to vary so that we may consider the case of shrinking sheets. The study of the flow of a fluid over a shrinking sheet has appeared much less in the literature than that of the corresponding stretching sheet problem, and in some special cases multiple solutions have been obtained. Some interesting works on shrinking sheet problems include [20]-33].

We show that for various physically meaningful values of the suction/injection parameter, the magnetic parameter and the viscoelastic parameter, multiple solutions may exist in either the stretching or shrinking sheet problem, while for other parametric values, we have no similarity solutions to the flow problem. Furthermore, we are able to prove that no more than two physically meaningful similarity solutions exist for all physical values of the parameters.

2. Formulation of the problem. Consider the flow of a second-order fluid obeying equations (11)-(4) past a flat sheet coinciding with the plane $y=0$, the flow being confined to $y>0$. Two equal and opposite forces are applied along the $x$-axis so that the wall is stretched, keeping the origin fixed, and a uniform magnetic field $B_{0}$ is imposed along the $y$-axis. The steady two-dimensional boundary layer equations for this fluid in the usual notation are

$$
\begin{gathered}
\frac{\partial u}{\partial x}+\frac{\partial v}{\partial y}=0 \\
u \frac{\partial u}{\partial x}+v \frac{\partial u}{\partial y}=\nu \frac{\partial^{2} u}{\partial y^{2}}-\frac{\sigma_{0} B_{0}^{2}}{\rho} u+\lambda\left\{\frac{\partial}{\partial x}\left(u \frac{\partial^{2} u}{\partial y^{2}}\right)-\frac{\partial u}{\partial y} \frac{\partial^{2} u}{\partial x \partial y}+v \frac{\partial^{3} u}{\partial y^{3}}\right\},
\end{gathered}
$$


where $\nu=\mu / \rho, \sigma_{0}$ is the electric conductivity, $B_{0}$ is the uniform magnetic field along the $y$-axis and $\lambda=\alpha_{1} / \rho$. In deriving these equations, it was assumed that the contribution due to the normal stress is of the same order of magnitude as that due to the shear stress (in addition to the usual boundary layer approximations). Thus both $\nu$ and $\lambda$ are $O\left(\delta^{2}\right)$, where $\delta$ is the boundary layer thickness. Also, the induced magnetic field is neglected (which is justified for a small magnetic Reynolds number; see [34). It is also assumed that the external electric field is zero and that the electric field due to polarization of charges is negligible.

The appropriate boundary conditions for the problem are given by

$$
\begin{gathered}
u=B x, v=-v_{0} \text { at } y=0, B \in \mathbb{R}, \\
u \rightarrow 0, \frac{\partial u}{\partial y} \rightarrow 0 \text { as } y \rightarrow \infty,
\end{gathered}
$$

where $\partial u / \partial y \rightarrow 0$ as $y \rightarrow \infty$ is the augmented condition. The sign of the parameter $B$ will determine if the sheet is stretching $(B>0)$ or shrinking $(B<0)$. Equations (5) (8) admit a self-similar solution of the form

$$
\begin{gathered}
u=|B| x f^{\prime}(\eta), v=-\sqrt{|B| \nu} f(\eta), \\
\eta=\sqrt{\frac{|B|}{\nu}} y,
\end{gathered}
$$

where the prime denotes differentiation with respect to $\eta$. Clearly, $u$ and $v$ defined above satisfy the continuity equation (5). Substituting equations (9) and (10) into equation (6) gives the similarity equation

$$
f^{\prime \prime \prime}(\eta)+f(\eta) f^{\prime \prime}(\eta)-\left(f^{\prime}(\eta)\right)^{2}=M f^{\prime}(\eta)+K\left[2 f^{\prime}(\eta) f^{\prime \prime \prime}(\eta)-\left(f^{\prime \prime}(\eta)\right)^{2}-f(\eta) f^{(i v)}(\eta)\right]
$$

subject to the boundary conditions

$$
f(0)=s, f^{\prime}(0)=\chi, \lim _{\eta \rightarrow \infty} f^{\prime}(\eta)=0, \lim _{\eta \rightarrow \infty} f^{\prime \prime}(\eta)=0,
$$

where $K=-\lambda B / \nu$ is the viscoelastic parameter and $M=\sigma_{0} B_{0}^{2} /(\rho B)$ is the magnetic parameter. We note that $s>0$ corresponds to suction at the surface, while $s<0$ corresponds to injection. Furthermore, $\chi=1$ corresponds to a stretching sheet, while $\chi=-1$ corresponds to a shrinking sheet. The magnetic parameter is always taken as $M \geq 0$, while various real values of the viscoelastic parameter $K$ have been considered. In Vajravelu and Rollins [18, the values $K \leq 0$ were considered for some special cases, in the event of $\chi=1$ (stretching sheet) and $s=0$. The study of solutions to the problem (11) - (12), particularly in the regions of the parametric space in which we have physically meaningful multiple solutions, shall be the focus of the present paper. Recall from 9] that physically meaningful scenarios occur when $K \leq 0$ (i.e., when $\lambda B / \nu \geq 0$ ), so we shall restrict ourselves to consideration of cases in which $M \geq 0$ and $K \leq 0$.

We remark that in similar stretching sheet problems where $s=K=M=0$, multiple solution branches have been found. See Liao [35, 36. Thus, it is not just in the shrinking sheet case where one may find nonuniqueness of the solutions. We will show that the boundary value problem (11)-(12) admits multiple solutions in the case of a stretching 
sheet $(\chi=1)$ with injection at the surface $(s<0)$ for certain values of the parameters. Furthermore, we show that in the event of a shrinking sheet, problem (11)-(12) may admit multiple solutions in either the suction $(s>0)$ or injection $(s<0)$ regimes.

3. Exact solutions. We obtain exact solutions to (11) which satisfy the boundary conditions given in (12). We discover that there may be no solutions, one solution, two solutions or three solutions, depending on the values of the physical parameters $s, \chi, K$ and $M$. Assume a solution to the nonlinear differential equation (11) of the form

$$
f(\eta)=A+B e^{-C \eta} .
$$

This assumption is often motivated by the result of Crane [37, where a rare exact solution to the Navier-Stokes equations,

$$
f(\eta)=1-e^{-\eta}
$$

was obtained when $s=M=K=0$ in (11)-(12), in the case of a stretching sheet $(\chi=1)$. From the first two boundary conditions in (12), we find that such a solution (13) must be of the form

$$
f(\eta)=s+\frac{\chi}{C}\left(1-e^{-C \eta}\right)
$$

while from the latter boundary conditions, we must have that $C>0$. Placing this solution expression into (11) yields the cubic equation

$$
K s C^{3}+(K \chi-1) C^{2}+s C+(\chi+M)=0 .
$$

That is, any constant $C$ in (14) must satisfy (15). Whether or not the solutions to (15) will be nonnegative reals depends on the values of the parameters $s, \chi, K$ and $M$. Indeed, we find that, depending on the values of these physical parameters, either zero or one or two solutions of (15) will give physically meaningful solutions of the form (14) to (11) - (12). We shall denote the solutions to [15] by $C_{1}, C_{2}$ and $C_{3}$ : defining the constants $\Lambda_{1}, \Lambda_{2}$ and $\Lambda_{3}$ by

$$
\begin{aligned}
\Lambda_{1}= & -72 K^{2} s^{2} \chi-36 K s^{2}-108 K^{2} s^{2} M-8 K^{3} \chi^{3}+24 K^{2} \chi^{2}-24 K \chi+8, \\
\Lambda_{2}= & -4 M-4 \chi+36 K^{2} s^{2} \chi M+4 K^{3} M \chi^{3}-12 K^{2} M \chi^{2}+12 K M \chi-s^{2}+4 K s^{4} \\
& +12 K \chi^{2}+8 K^{2} s^{2} \chi^{2}+20 K s^{2} \chi+4 K^{3} \chi^{4}-12 K^{2} \chi^{3}+18 K s^{2} M+27 K^{2} s^{2} M^{2}, \\
\Lambda_{3}= & -3 K s^{2}+K^{2} \chi^{2}-2 K \chi+1,
\end{aligned}
$$


the solutions $C_{1}, C_{2}$ and $C_{3}$ to the equation (15) are explicitly given by

$$
\begin{aligned}
C_{1}= & \frac{1}{6 K s}\left(\Lambda_{1}+12 \sqrt{3 \Lambda_{2}} K s\right)^{1 / 3}+\frac{2 \Lambda_{3}}{3}\left(\Lambda_{1} K s+12 \sqrt{3 \Lambda_{2}} K s\right)^{1 / 3}-\frac{K \chi-1}{3 K s}, \\
C_{2}= & \frac{\sqrt{3} i-1}{12 K s}\left(\Lambda_{1}+12 \sqrt{3 \Lambda_{2}} K s\right)^{1 / 3} \\
& +\frac{(\sqrt{3} i-1) \Lambda_{3}}{3}\left(\Lambda_{1} K s+12 \sqrt{3 \Lambda_{2}} K s\right)^{1 / 3}-\frac{K \chi-1}{3 K s}, \\
C_{3}= & -\frac{\sqrt{3} i-1}{12 K s}\left(\Lambda_{1}+12 \sqrt{3 \Lambda_{2}} K s\right)^{1 / 3} \\
& -\frac{(\sqrt{3} i-1) \Lambda_{3}}{3}\left(\Lambda_{1} K s+12 \sqrt{3 \Lambda_{2}} K s\right)^{1 / 3}-\frac{K \chi-1}{3 K s},
\end{aligned}
$$

where $i^{2}=-1$. These solutions are clearly not valid for $K s=0$ (indeed, in such a case, (15) reduces to a quadratic equation in $C$ ). Hence we shall consider the cases in which $s=0$ and $K=0$, below separately.

3.1. Explicit exact solutions in some special cases. We may consider a number of special case solutions which do not require solutions to the cubic equation (15). In the special case $\chi=0$, we have from (14) that

$$
f(\eta)=s .
$$

3.1.1. The case when $s=0$. When $s=0$, we have the single physically meaningful solution

$$
f(\eta)=\frac{\chi}{C}\left(1-e^{-C \eta}\right), \quad \text { where } \quad C=\sqrt{\frac{1+M}{1-K \chi}},
$$

which is valid for $K \chi<1$. In the special case of $s=0$ and $\chi=1$, we obtain

$$
f(\eta)=\sqrt{\frac{1-K}{1+M}}\left(1-\exp \left\{-\sqrt{\frac{1+M}{1-K}} \eta\right\}\right),
$$

a monotonically increasing positive function over $\eta \in(0, \infty)$ valid for all $M \geq 0$ and all $-1<K \leq 0$. In the case of $M=0$, a corresponding solution was obtained by Troy et al. [13] and Vajravelu and Rollins [18, while Abel and Nandeppanavar [38] obtained (20) for $M \geq 0$.

When $s=0$ and $\chi=-1$, we obtain

$$
f(\eta)=-\sqrt{\frac{1+K}{1+M}}\left(1-\exp \left\{-\sqrt{\frac{1+M}{1+K}} \eta\right\}\right),
$$

a monotonically decreasing negative function over $\eta \in(0, \infty)$ valid for all $M \geq 0$ and all $-1<K \leq 0$.

3.1.2. The case when $K=0$. This case is well studied in the literature in the case of a stretching sheet (see, for instance, Wang [39]). When $K=0$, we have possible solutions of the form

$$
f_{ \pm}(\eta)=s+\frac{\chi}{C_{ \pm}}\left(1-e^{-C_{ \pm} \eta}\right), \quad \text { where } \quad C_{ \pm}=\frac{s \pm \sqrt{s^{2}+4(\chi+M)}}{2} .
$$


The special case of Eq. (22) for a stretching sheet $(\chi=1)$ has been obtained by Chakrabarti and Gupta [40] and others (see, for instance, [41-443]). In this case, the positive root, $C_{+}$, is taken as the only physically meaningful solution. In the case of a shrinking sheet (i.e., $\chi=-1)$ and in the absence of a magnetic field $(M=0)$, Eq. (22) reduces to the result of Miklavcic and Wang [31, where it is shown that there are two solutions for any $s>2$ and there is one solution for $s=2$. No solution exists for $s<2$. In the case of a shrinking sheet with magnetic term $M>0$, Eq. (22) agrees with the results given in Fang and Zhang 20, where both $C_{+}$and $C_{-}$may correspond to physically meaningful solutions, for certain values of the parameters $s$ and $M$. In particular, for $0<M<1$, there are two solution branches corresponding to $C_{+}$and $C_{-}$in Eq. (22) which exist only for mass suction from the surface $(s>0)$, such that $s$ satisfies $s^{2}>4(\chi+M)$. Note that for the $C_{+}$-branch, the wall shear stress increases with the increase of the mass suction and magnetic parameter. However, for the $C_{-}$-branch, the wall shear stress decreases with the increase of the mass suction and magnetic parameters. When $M=1$ there exists only one solution when there is suction from the surface $(s>0)$, and the wall shear stress for the solution corresponding to $M=1$ increases with increasing mass suction parameter $s$. For $M>1$, there is only one solution, corresponding to the $C_{+}$-branch; this solution exists for both mass suction $(s>0)$ and mass injection $(s<0)$ (i.e., for all $s \in \mathbb{R}$ ), which also shows that the wall shear stress increases with the mass suction parameter.

The MHD flow over a shrinking sheet is also greatly different from the MHD flow over a stretching sheet for Newtonian fluids (see [40-443) and for non-Newtonian fluids (see, for instance, Liao [4]). Thus, the shrinking sheet MHD flow shows richer nonlinear behavior in the solution domain, with possible multiple solutions for many physically meaningful values of the model parameters.

Aside from the exponentially decaying solutions which are the focus of the present paper, we remark that there is an algebraically decaying solution

$$
f(\eta)=\frac{6}{\eta+\sqrt{6}}
$$

in the shrinking sheet case, when $s=\sqrt{6}$ and $M=0$ (see Fang and Zhang [20]).

If $K=0$ and $s<0$, then the solution $f_{+}$is physically meaningful provided $\chi>-M$, while the solution $f_{-}$is not physically meaningful. However, if $\chi<-M$ when $K=0$ and $s<0$, neither of $f_{ \pm}$are physically meaningful. If $K=0$ and $s>0$, then $f_{+}$ is a physically meaningful solution provided $\chi>-M-s^{2} / 4$ and $f_{-}$is a physically meaningful solution provided $\chi<-M$. Thus, when $K=0$ and $s>0$, there is always one physically meaningful solution when $\chi<-M-s^{2} / 4$ or $\chi>-M$ while there are always two physically meaningful solutions when $-M-s^{2} / 4<\chi<-M$.

3.1.3. The case when $\chi=-1$ and $M=1$. When $\chi=-1$ and $M=1$, we find that there is one physically meaningful solution of the form (14) for all $K<0$ and all $s \neq 0$ given by

$$
f(\eta)=s-\frac{1}{C}\left(1-e^{-C \eta}\right), \quad \text { where } \quad C=\frac{K+1}{2 K s}+\frac{1}{2} \sqrt{\left(\frac{K+1}{K s}\right)^{2}-\frac{4}{K}} .
$$


This results from the fact that the cubic equation (15) reduces to a quadratic equation in $C$, as the constant term in (15) vanishes in this special case (as $\chi+M=-1+1=0$ ).

3.2. The nonexistence of three physically meaningful solutions. We remark that while we obtain either no solution, one solution, or two solutions in a number of cases, there are no cases in which there will be three solutions for physically meaningful parameters. The cubic equation in $C$ defined by (15) has in general two critical points, say $Y_{ \pm}$, defined by the two solutions to the quadratic equation

$$
3 K s Y^{2}+2(K \chi-1) Y+s=0 .
$$

In order for the cubic equation in $C$ defined by (15) to have three positive solutions, it is necessary for (24) to have two positive solutions; that is to say, we must have $Y_{ \pm}>0$. Explicitly,

$$
Y_{ \pm}=\frac{-(K \chi-1) \pm \sqrt{(K \chi-1)^{2}-3 K s^{2}}}{3 K s}
$$

so in order for $Y_{ \pm}>0$ to hold, either of the following conditions must be satisfied:

(i) $K \chi-1<0, K s>0, s>0,(K \chi-1)^{2}-3 K s^{2}>0$, or

(ii) $K \chi-1>0, K s<0, s<0,(K \chi-1)^{2}-3 K s^{2}>0$.

Notice that $K s>0$ and $s>0$ imply that $K>0$, while $K s<0$ and $s<0$ also imply that $K>0$. Yet, for a physical solution, we must have $K \leq 0$. As such, when $K \leq 0$, it will not be true that $Y_{ \pm}>0$ and therefore the cubic equation in $C$ defined by (15) will never have three positive roots corresponding to three physically meaningful solutions. Graphically, we depict this scenario in Figure 7, we observe three positive solutions for $C$ in the nonphysical regime of $K>0$, while in the physical regime $K \leq 0$ we observe no more than two positive solutions of the form (14).

4. Discussion of the results. As discussed in Vajravelu and Rollins [18, there is only one physically meaningful solution in the case of a stretching sheet $(\chi=1)$ with suction $(s>0)$, for $M \geq 0$ and $K \leq 0$. However, we find that this is not true for a stretching sheet with injection $(s<0)$, or for a shrinking sheet $(\chi=-1)$ in general. Which of the exact solutions actually occurs will depend upon the flow stability, which we do not investigate in this paper. We remark that, in the case of dual solutions, the exponential decay rate of each solution (the value of $C$ for each) will differ; as such, the value of the shear stress at the wall, $f^{\prime \prime}(0)$, will differ between the dual solutions. We now discuss the influence of the parametric values of $s, K$ and $M$ on the existence of similarity solutions of the form prescribed in (14).

In Figures 14, we observe the influence of the suction/injection parameter $s \in \mathbb{R}$ on the number of solutions. In the case of a stretching sheet, we observe one physically meaningful solution of the form (14) when there is suction $(s>0)$. Furthermore, the decay rate of this solution increases with an increase in $s$ when $0 \leq M \leq 1$, and decreases with an increase in $s$ when $M>1$. In the case of a stretching sheet with injection $(s<0)$, we observe dual solutions when $0 \leq M \leq 1$, and either zero, one or two solutions when $M>1$. In the latter case, we denote the values of $s$ admitting one solution by $s_{1}^{*}$ and $s_{2}^{*}$, where $s_{1}^{*}<s_{2}^{*}$; between these values there are no physical solutions. When $0 \leq M \leq 1$, the decay rates of both of the dual solutions are increasing with an increase in $s$, while 
when $M>1$ the decay rate of one solution increases while the other decay rate decreases, given an increase in $s$. In the case of the shrinking sheet, let us first consider suction $(s>0)$, where we find either zero, one or two solutions in the case that $0 \leq M \leq 1$ (where one of the dual solutions has decreasing decay rate with an increase in $s$ and the other dual solution has an increasing decay rate with an increase in $s$ ), or a single solution when $M>1$ (where this single solution has a decreasing decay rate for an increase in $s$ ). When we consider a shrinking sheet with injection $(s<0)$, we observe a single solution when $0 \leq M \leq 1$ (which has increasing exponential decay rate as $s$ increases) and either zero, one or two solutions when $M>1$ (with one of the dual solutions having decreasing decay rate with an increase in $s$ and the other solution having increasing decay rate with an increase in $s$ ). Table 1 summarizes the number of solutions of the form (14) found as we consider various values of $s \in \mathbb{R}$ for fixed values of the other parameters.

Observe that for both the stretching and shrinking sheet problems there may exist no solutions of the form (14) in the case of injection from the surface $(s<0)$, for sufficiently large negative $K$. As shown in Figures 6 and 8 , in the case of injection from the surface (in either the stretching or shrinking sheet problem) there exists some $K^{*}$ such that for all $K \in\left(K^{*}, 0\right]$ there exist two physically meaningful solutions of the form (14). However, for all $K \in\left(-\infty, K^{*}\right)$, there are no solutions of the form (14) to the boundary value problem (11) -(12). When $K=K^{*}<0$, there exists a unique solution of the form (14) to (11) -(12), in the case of injection for either the stretching or shrinking sheet problems. In the case of suction from the surface $(s>0)$, we see that there appears to exist at least one solution for either the case of a stretching or shrinking sheet for all physical values of $K \leq 0$. This is shown in Figure 2, where we consider the influence of the viscoelastic parameter $K \leq 0$ on the obtained decay rates $C$ for the solutions of the form (14), in the case of suction $(s>0)$ and injection $(s<0)$ from the surface, for both the stretching and shrinking sheet problems. Note that for the stretching sheet problem with suction $(s>0)$, we obtain the result of Vajravelu and Rollins [18: There exists only one solution of the form (14) for the stretching problem in the case of suction from the stretching surface for all $M \geq 0$, given physically meaningful values of the viscoelastic parameter $K$. In the case of a shrinking sheet, however, we observe two meaningful solutions in the case of suction. These findings are summarized in Table 2, while the four standard cases are considered in Figures 58

When we consider the stretching sheet problem with suction at the stretching surface, we recover the results of Vajravelu and Rollins [18, where it was shown that there exists a single physically meaningful solution of the form (14) when $\chi=1$ and $s>0$, for any fixed $M \geq 0$ (assuming, of course, physically meaningful values of $K$; that is, $K \leq 0$ ). This is in contrast to the case of a stretching sheet with injection from the surface, where there exists some $M^{*}>0$ such that for all $M \in\left[0, M^{*}\right)$ there exist two physically meaningful solutions of the form (14) to (11)-(12) and no physically meaningful solutions when $M \in\left(M^{*}, \infty\right)$. When $M=M^{*}$ for the stretching sheet problem with injection at the surface, there exists a unique solution of the form (14) to the boundary value problem (11)-(12). In the case of a shrinking sheet, the region of $M \in[0, \infty)$ admitting a fixed number of solutions becomes more complicated; we remark that these findings are summarized in Table 3, In particular, we find that for the shrinking sheet problem with 
suction from the surface, there exists $M^{*}>0$ such that for all $M \in\left[0, M^{*}\right)$ there exist no solutions of the form (14), while for all $M \in\left(M^{*}, 1\right)$ there exist two solutions of the form (14). There exists a unique solution of the form (14) to the shrinking sheet problem (with suction from the shrinking surface) when either $M \geq 1$ or $M=M^{*}$. However, for the shrinking sheet problem with injection from the shrinking surface, we find that there exists $M^{*}>0$ such that for all $M \in[0,1]$ there exists one physically meaningful solution of the form (14), while for all $M \in\left(1, M^{*}\right)$ there exist two physical solutions of the form (14). Furthermore, for a shrinking sheet with injection at the surface, there exist no physical solutions of the form (14) when $M>M^{*}$. Notice that $M=1$ always provides a unique solution of the form (14) in the case of a shrinking sheet, regardless of whether there is suction or injection at the surface (or, neither, i.e. $s=0$ ); this unique solution decay rate corresponding to $M=1$ is shown prominently in Figures 11,12 and follows from the special case considered in Eq. (23).

In Figure 13, we provide the profiles of $f(\eta)$ and $f^{\prime}(\eta)$ for several sets of values of the parameters (see Table 4), to demonstrate cases in which there are single or dual solutions in the stretching sheet problem $(\chi=1)$. Likewise, we present the profiles of $f(\eta)$ and $f^{\prime}(\eta)$ for several sets of values of the physical parameters for the shrinking sheet problem $(\chi=-1)$ in Figure 14. Parameter values selected in each of these figures are given in Table 4. In those cases where multiple solutions exist, we note that the decay rates of each solution will differ (as the decay rates are given by the value of $C$ in the solution to (15)). Thus, in computing the shear stress at the surface, $f^{\prime \prime}(0)$, we find that there will be two such values in the case of two physically meaningful values $C_{I}>0$ and $C_{I I}>0$; these are given by $f_{I}^{\prime \prime}(0)=-C_{I} \chi$ and $f_{I I}^{\prime \prime}(0)=-C_{I I} \chi$. As expected in the case of a stretching sheet, in the event of multiple solutions we have that $f_{I}^{\prime \prime}(0)=-C_{I}<0$ and $f_{I I}^{\prime \prime}(0)=-C_{I I}<0$. Similarly, in the shrinking sheet problem, for any given multiple solutions corresponding to $C_{I}>0$ and $C_{I I}>0$, we have that $f_{I}^{\prime \prime}(0)=C_{I}>0$ and $f_{I I}^{\prime \prime}(0)=C_{I I}>0$.

Future work may involve a similar analysis for higher-order fluids. We may attempt to determine what other physical fluid flow scenarios admit self-similar solutions of the form (14). Understanding the existence and uniqueness (or nonuniqueness) of such solutions for general non-Newtonian fluids is worth studying.

\begin{tabular}{|c|c|c|c|}
\hline & \multicolumn{3}{|c|}{ Values of $s$ Giving Physically Meaningful Solutions } \\
\hline Parameters & No Solutions & One Solution & Two Solutions \\
\hline$K=-1, M=0.5, \chi=1$ & - & $s \geq 0$ & $s<0$ \\
\hline$K=-1, M=1.5, \chi=1$ & $s_{1}^{*}<s<s_{2}^{*}$ & $s=s_{1}^{*}, s=s_{2}^{*}, s \geq 0$ & $s<s_{1}^{*}, s_{2}^{*}<s<0$ \\
\hline$K=-1, M=0.5, \chi=-1$ & $0 \leq s<s^{*}$ & $s<0, s=s^{*}$ & $s>s^{*}$ \\
\hline$K=-1, M=1.5, \chi=-1$ & $s^{*}<s \leq 0$ & $s=s^{*}, s>0$ & $s<s^{*}$ \\
\hline
\end{tabular}

TABLE 1. Number of physically meaningful solutions for various values of $s \in \mathbb{R}$. 


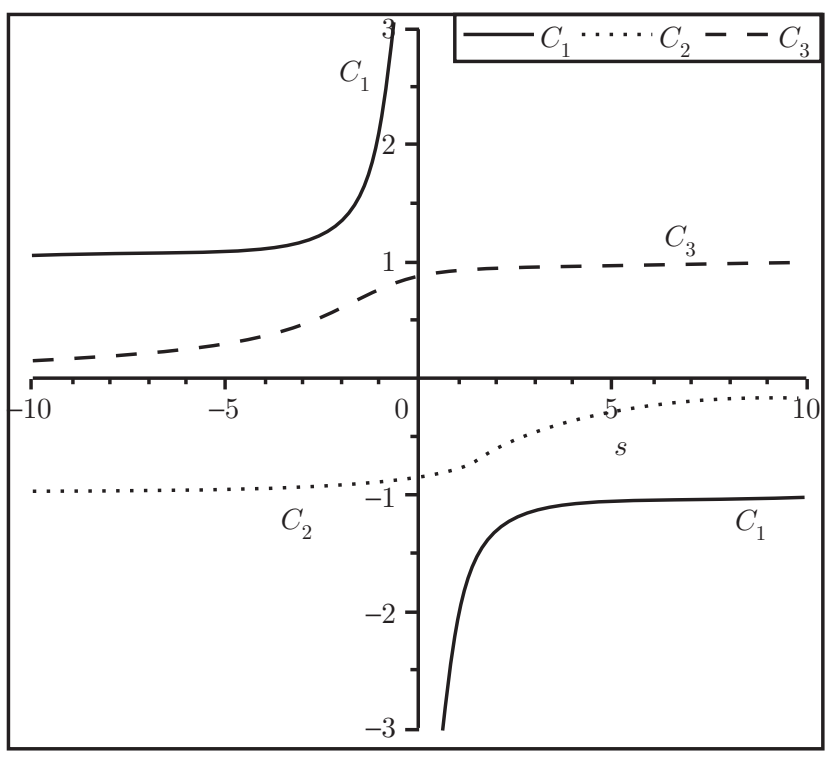

Fig. 1. $C$-curves. Physically meaningful solutions correspond to $C>$ 0 . Curves are plotted for various values of the suction/injection parameter $s$ when $K=-1, M=0.5$ and $\chi=1$.

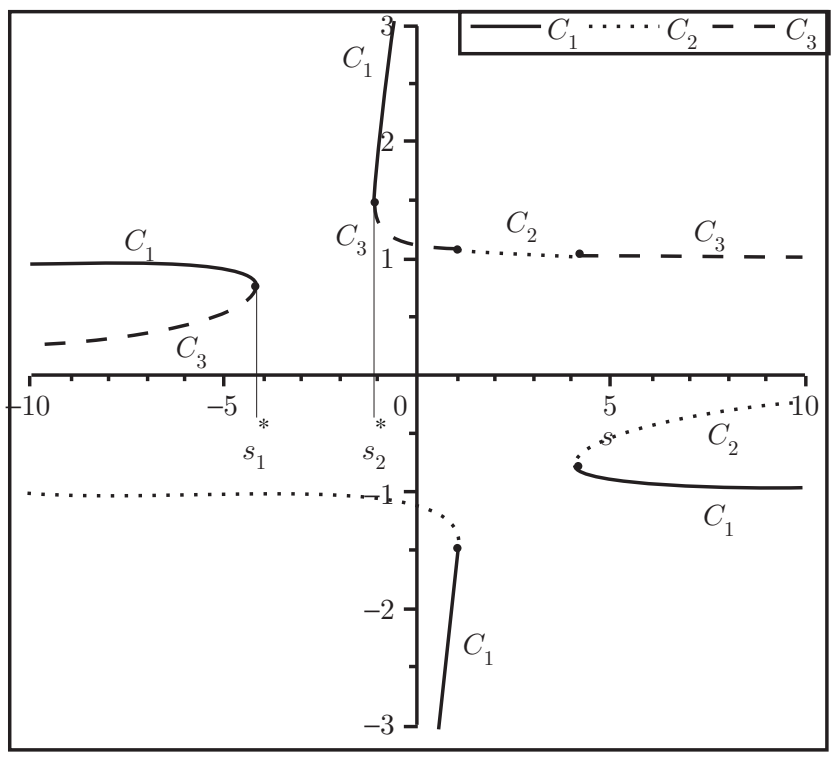

FIG. 2. $C$-curves. Physically meaningful solutions correspond to $C>$ 0 . Curves are plotted for various values of the suction/injection parameter $s$, when $K=-1, M=1.5$ and $\chi=1$. 


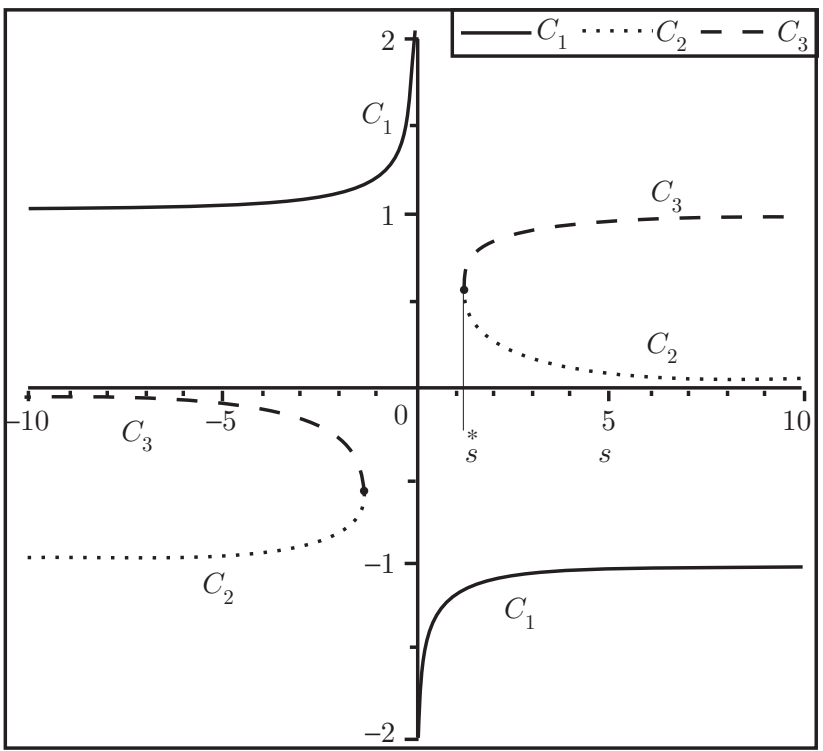

Fig. 3. $C$-curves. Physically meaningful solutions correspond to $C>$ 0 . Curves are plotted for various values of the suction/injection parameter $s$, when $K=-1, M=0.5$ and $\chi=-1$.

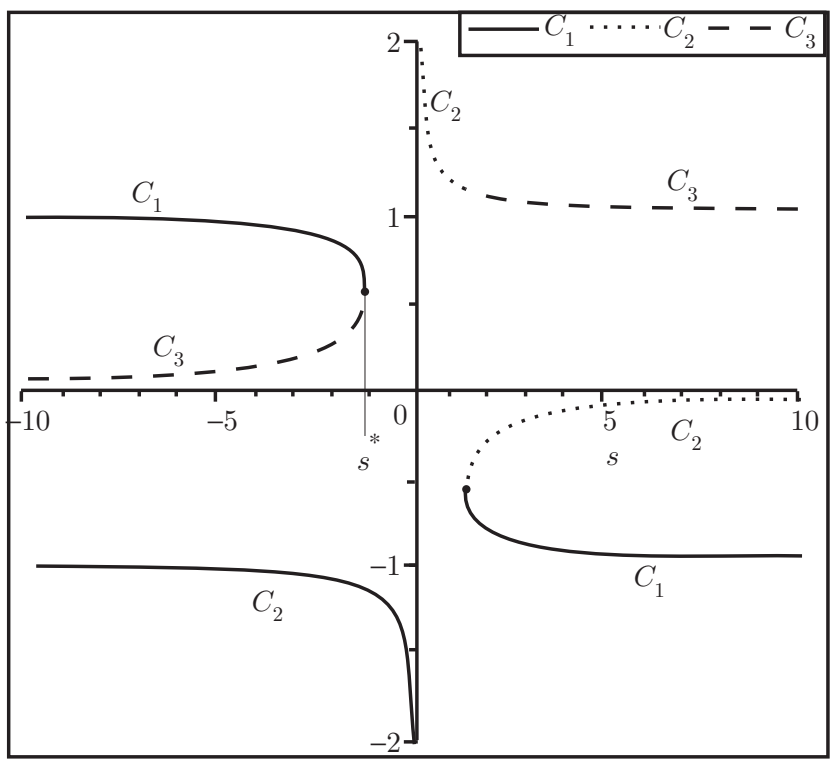

FIG. 4. $C$-curves. Physically meaningful solutions correspond to $C>$ 0 . Curves are plotted for various values of the suction/injection parameter $s$, when $K=-1, M=1.5$ and $\chi=-1$. 


\begin{tabular}{|c|c|c|c|}
\hline & \multicolumn{2}{|c|}{ Values of $K \leq 0$ Giving Physically Meaningful Solutions } \\
\hline Parameters & No Solutions & One Solution & Two Solutions \\
\hline$s=1, M=1.1, \chi=1$ & - & $K \leq 0$ & - \\
\hline$s=-1, M=1.1, \chi=1$ & $K<K^{*}$ & $K=K^{*}$ & $K^{*}<K \leq 0$ \\
\hline$s=1, M=1.1, \chi=-1$ & - & - & $K \leq 0$ \\
\hline$s=-1, M=1.2, \chi=-1$ & $K<K^{*}$ & $K=K^{*}$ & $K^{*}<K \leq 0$ \\
\hline
\end{tabular}

TABLE 2. Number of physically meaningful solutions for various values of $K \leq 0$.

\begin{tabular}{|c|c|c|c|}
\hline & \multicolumn{3}{|c|}{ Values of $M \geq 0$ Giving Physically Meaningful Solutions } \\
\hline Parameters & No Solutions & One Solution & Two Solutions \\
\hline$s=1, K=-1, \chi=1$ & - & $M \geq 0$ & - \\
\hline$s=-1, K=-1, \chi=1$ & $M>M^{*}$ & $M=M^{*}$ & $0 \leq M<M^{*}$ \\
\hline$s=1, K=-1, \chi=-1$ & $0 \leq M<M^{*}$ & $M=M^{*}, M \geq 1$ & $M^{*}<M<1$ \\
\hline$s=-1, K=-1, \chi=-1$ & $M>M^{*}$ & $M=M^{*}, 0 \leq M \leq 1$ & $1<M<M^{*}$ \\
\hline
\end{tabular}

TABLE 3. Number of physically meaningful solutions for various values of $M \geq 0$.

\begin{tabular}{|c|c|c|c|c|c|}
\hline & $\chi$ & $s$ & $K$ & $M$ & Physical values of $C$ \\
\hline I & 1 & -2 & -1 & 0.5 & (a) $0.6053779405 ;$ (b) 1.327721191 \\
\hline II & 1 & 2 & -1 & 0.5 & 0.9330991312 \\
\hline III & 1 & -7 & -1 & 1.5 & (a) $0.3683562397 ;$ (b) 0.9442071777 \\
\hline IV & 1 & -1 & -1 & 1.5 & (a) $1.315448807 ;$ (b) 1.762713780 \\
\hline V & 1 & 2 & -1 & 1.5 & 1.058971136 \\
\hline VI & -1 & -2 & -1 & 0.5 & 1.107159872 \\
\hline VII & -1 & 3 & -1 & 0.5 & (a) $0.1717313046 ;$ (b) 0.9030131417 \\
\hline VIII & -1 & -5 & -1 & 1.5 & (a) $0.1010312579 ;(b) 0.9456492739$ \\
\hline IX & -1 & 2 & -1 & 1.5 & 1.107159872 \\
\hline
\end{tabular}

TABle 4. Physical values of decay rate $C$ of solutions given fixed parameter values of $\chi, s, K$ and $M$. 


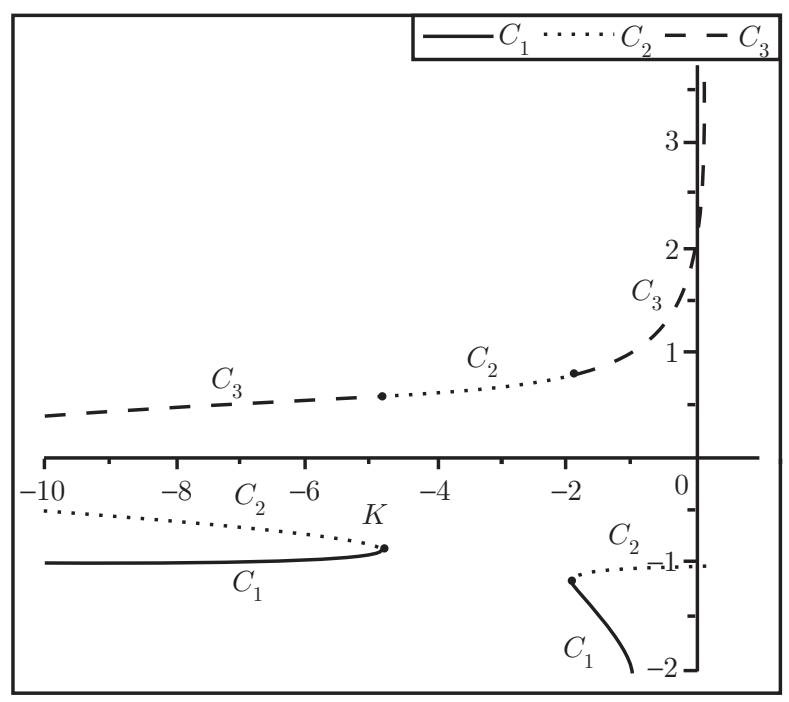

Fig. 5. $C$-curves. Physically meaningful solutions correspond to $C>$ 0 . Curves are plotted for various values of the viscoelastic parameter $K$, when $s=1, M=1.1$ and $\chi=1$.

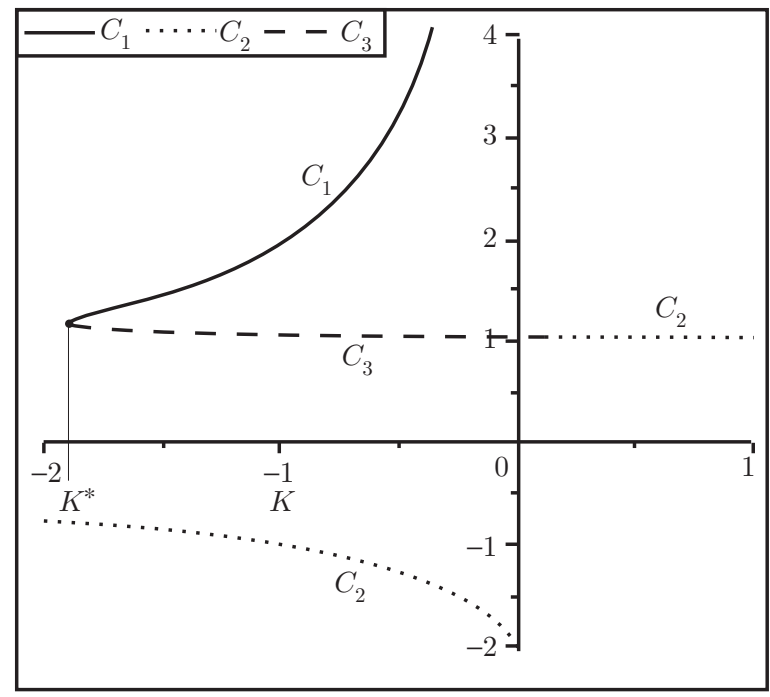

FIG. 6. $C$-curves. Physically meaningful solutions correspond to $C>$ 0 . Curves are plotted for various values of the viscoelastic parameter $K$, when $s=-1, M=1.1$ and $\chi=1$. 


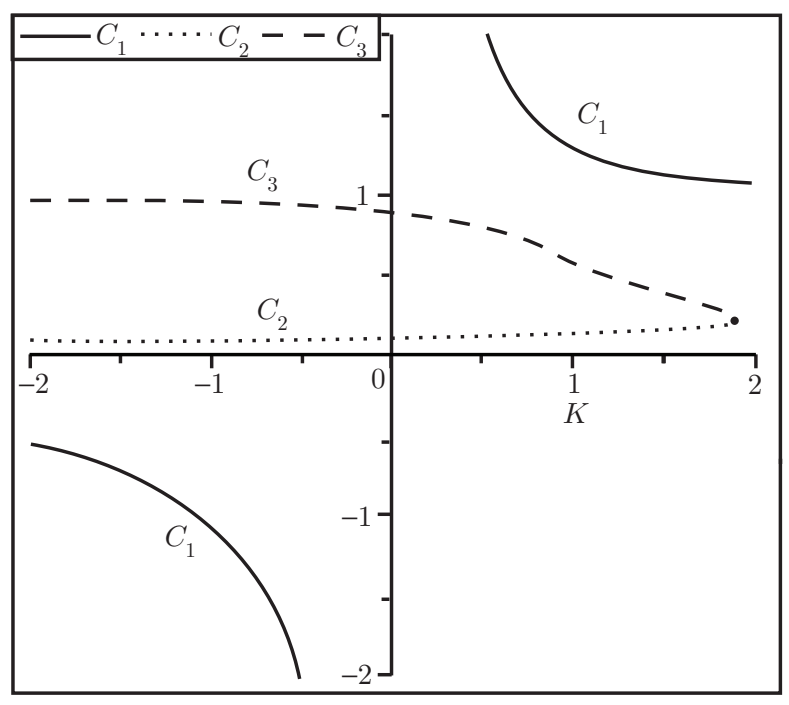

Fig. 7. $C$-curves. Physically meaningful solutions correspond to $C>$ 0 . Curves are plotted for various values of the viscoelastic parameter $K$, when $s=1, M=1.1$ and $\chi=-1$.

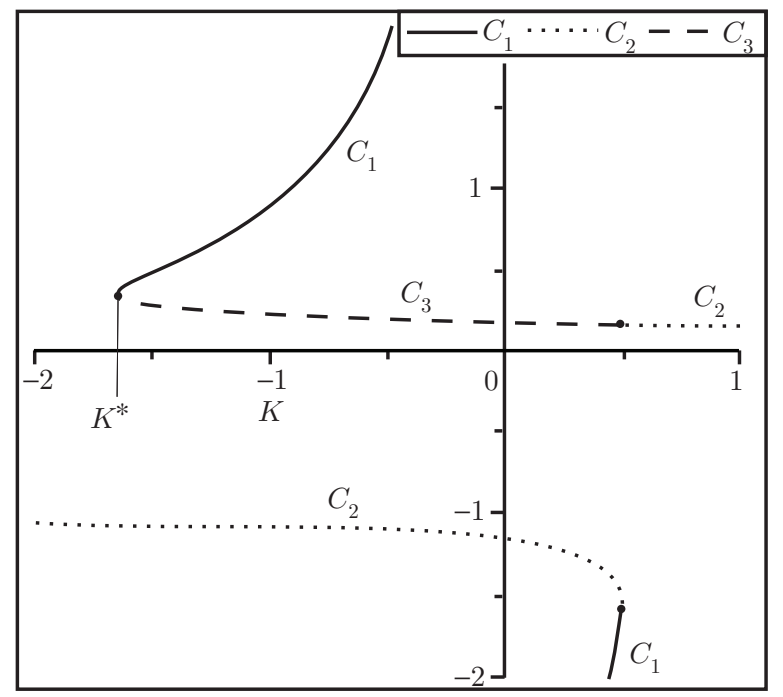

FIG. 8. $C$-curves. Physically meaningful solutions correspond to $C>$ 0 . Curves are plotted for various values of the viscoelastic parameter $K$, when $s=-1, M=1.2$ and $\chi=-1$. 


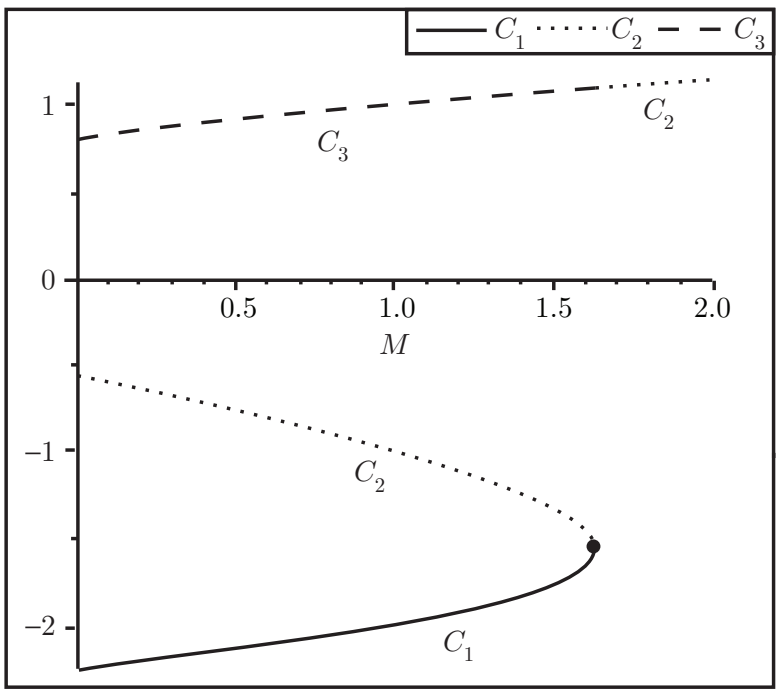

Fig. 9. $C$-curves. Physically meaningful solutions correspond to $C>$ 0 . Curves are plotted for various values of the magnetic parameter $M$, when $s=1, K=-1$ and $\chi=1$.

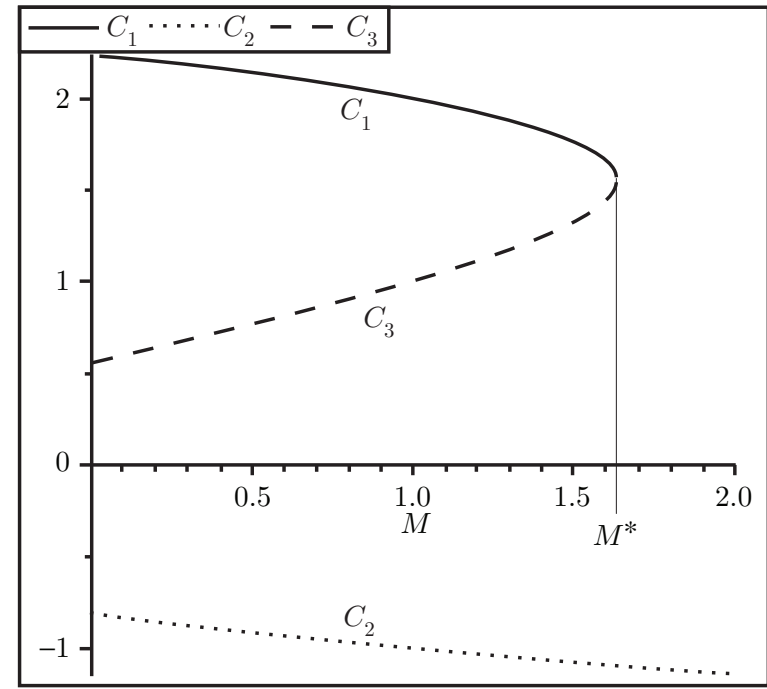

FIG. 10. $C$-curves. Physically meaningful solutions correspond to $C>0$. Curves are plotted for various values of the magnetic parameter $M$, when $s=-1, K=-1$ and $\chi=1$. 


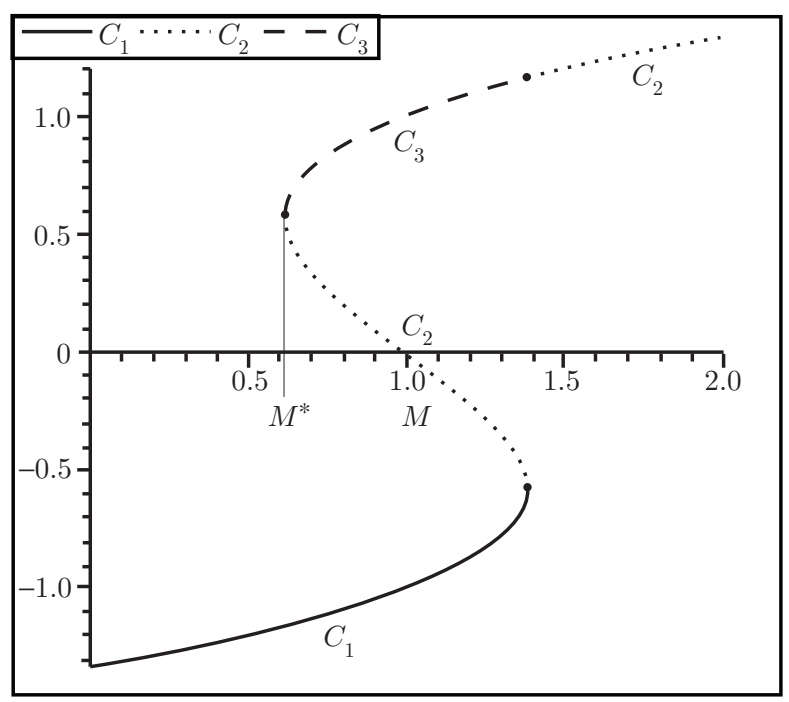

Fig. 11. $C$-curves. Physically meaningful solutions correspond to $C>0$. Curves are plotted for various values of the magnetic parameter $M$, when $s=1, K=-1$ and $\chi=-1$.

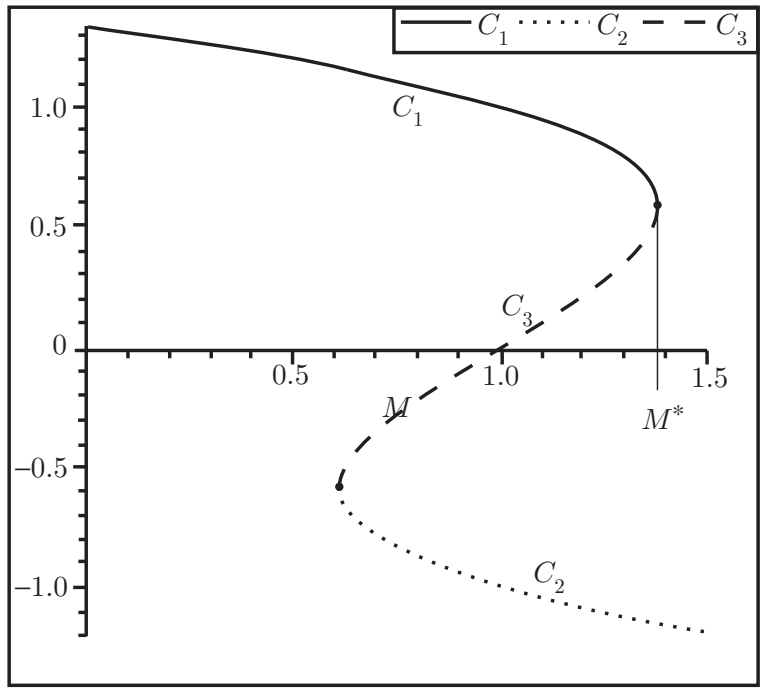

FIG. 12. $C$-curves. Physically meaningful solutions correspond to $C>0$. Curves are plotted for various values of the magnetic parameter $M$, when $s=-1, K=-1$ and $\chi=-1$. 


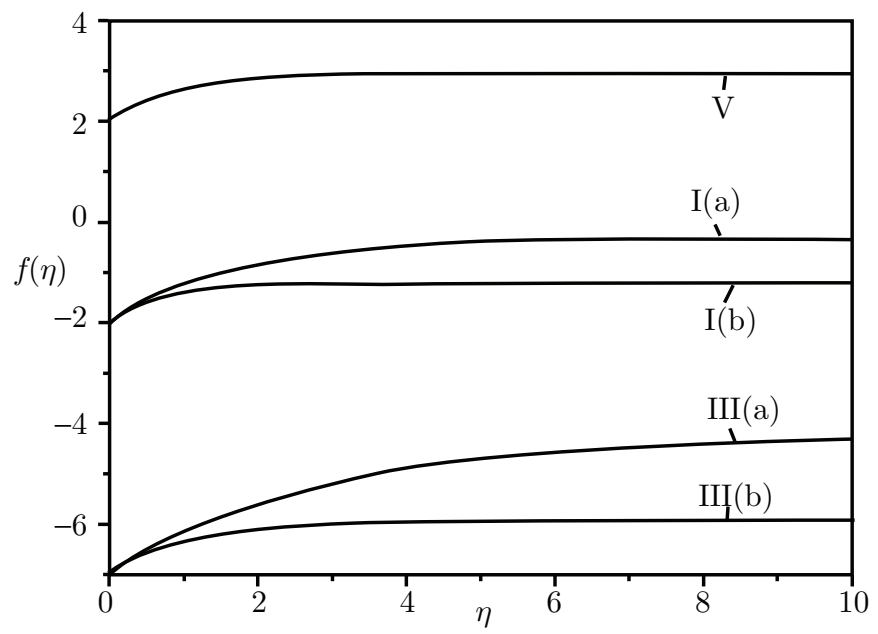

FIG. 13. Profiles for $f(\eta)$ in the case of a stretching sheet $(\chi=1)$. Parameter values are as given in Table 4 Observe the multiple solutions for $s=-2$ and $s=-7$.

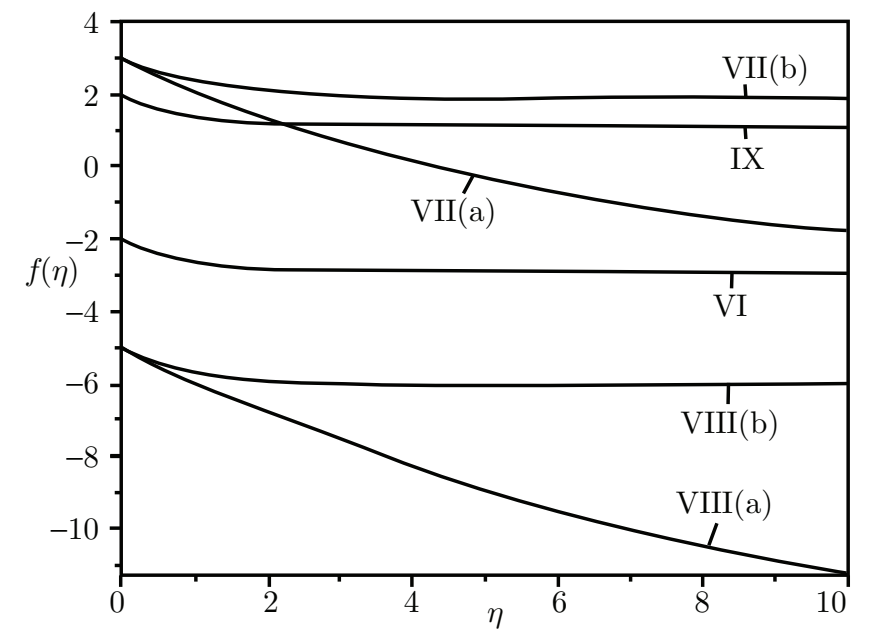

Fig. 14. Profiles for $f(\eta)$ in the case of a shrinking sheet $(\chi=-1)$. Parameter values are as given in Table 4 Observe the multiple solutions for $s=3$ and $s=-5$. 


\section{REFERENCES}

[1] C. Truesdell, W. Noll, The non-linear field theories of mechanics, in: S. Flugge (Ed.), Encyclopedia of Physics, III/3, Springer, Berlin, 1965, pp. 1-591. MR0193816 (33:2030)

[2] K.R. Rajagopal, On boundary conditions for fluids of the differential type, in: A. Sequeira (Ed.), Navier-Stokes Equations and Related Nonlinear Problems, Plenum Press, New York, 1995, pp. 273-278. MR:1373221

[3] K.R. Rajagopal, P.N. Kaloni, Some remarks on boundary conditions for fluids of the differential type, in: G.A.C. Graham, S.K. Malik (Eds.), Continuum Mechanics and its Applications, Hemisphere, New York, 1989, pp. 935-942. MR1051699

[4] K.R. Rajagopal, A.S. Gupta, An exact solution for the flow of a non-Newtonian fluid past an infinite plate, Meccanica 19 (1984) 158-160. MR767046 (85k:76008)

[5] D.W. Beard, K. Walters, Elastico-viscous boundary layer flows, Proc. Camb. Phil. Soc. 60 (1964) 667-674. MR0171475 (30:1706)

[6] V.K. Garg, K.R. Rajagopal, Flow of a non-Newtonian fluid past a wedge, Acta Mechanica 88 (1991) 113-123. MR.1111096

[7] G.K. Rajeswari, S.L. Rathna, Flow of a particular class of non-Newtonian visco-elastic and viscoelastic fluids near a stagnation point, Z. Angew. Math. Phys. 13 (1962) 43-57. MR0141330(25:4736)

[8] R.S. Rivlin, J.L. Ericksen, Stress deformation relations for isotropic materials, J. Rat. Mech. Anal. 4 (1955) 323-425. MR0068413 (16:881a)

[9] J.E. Dunn, K.R. Rajagopal, Fluids of differential type: Critical review and thermodynamic analysis, Int. J. Engrg. Sci. 33 (1995) 689-729. MR1321925 (96a:76006)

[10] K.R. Rajagopal, A.S. Gupta, T.A. Na, A note on the Falkner-Skan flows of a non-Newtonian fluid, Int. J. Non-Linear Mech. 18 (1983) 313-320. MR718753 (85a:76024)

[11] K. Vajravelu, D. Rollins, Heat transfer in a viscoelastic fluid over a stretching sheet, J. Math. Anal. Appl. 158 (1991) 241-255. MR.1113413 (92b:76003)

[12] M.S. Sarma, B.N. Rao, Heat transfer in a viscoelastic fluid over a stretching sheet, J. Math. Anal. Appl. 222 (1998) 268-275. MR.1623919 (99b:76005)

[13] W.C. Troy, E.A. Overman, G.B. Ermentrout, J.P. Keener, Uniqueness of flow of a second-order fluid past a stretching sheet, Quart. Appl. Math. 44 (1987) 753-755. MR.872826 (87m:76009)

[14] W.D. Chang, The nonuniqueness of the flow of a viscoelastic fluid over a stretching sheet, Quart. Appl. Math. 47 (1989) 365-366. MR998108 (90g:76024)

[15] P.S. Lawrence, B.N. Rao, Reinvestigation of the nonuniqueness of the flow of a viscoelastic fluid over a stretching sheet, Quart. Appl. Math. 51 (1993) 401-404. MR.1233521 (94e:76002)

[16] W.D. Chang, N.D. Kazarinoff, C. Lu, A new family of explicit solutions for the similarity equations modelling flow of a non-Newtonian fluid over a stretching sheet, Arch. Rat. Mech. Anal. 113 (1991) 191-195. MR.1079187 (91k:76009)

[17] K. Vajravelu, T. Roper, Flow and heat transfer in a second grade fluid over a stretching sheet, Int. J. Non-Linear Mech. 34 (1999) 1031-1036.

[18] K. Vajravelu, D. Rollins, Hydromagnetic flow of a second grade fluid over a stretching sheet, Applied Mathematics and Computation 148 (2004) 783-791. MR2024543

[19] A.D. Barinberg, A.B. Kapusta, B.V. Chekin, Magnitnaya Gidrodinamika (English translation) 11 (1975) 111-121.

[20] T. Fang, J. Zhang, Closed-form exact solutions of MHD viscous flow over a shrinking sheet, Commun. Nonlinear Sci. Numer. Simulat. 14 (2009) 2853-2857.

[21] T. Fang, J. Zhang, S. Yao, Viscous Flow over an Unsteady Shrinking Sheet with Mass Transfer, Chinese Phys. Lett. 26 (2009) 014703.

[22] T. Fang, Boundary layer flow over a shrinking sheet with power-law velocity, International Journal of Heat and Mass Transfer 51 (2008) 5838-5843.

[23] T. Fang, W. Liang, C. F. Lee, A new solution branch for the Blasius equation - A shrinking sheet problem, Computers and Mathematics with Applications 56 (2008) 3088-3095. MR2474564 (2009k:76054)

[24] T. Hayat, Z. Abbas, T. Javed, M. Sajid, Three-dimensional rotating flow induced by a shrinking sheet for suction, Chaos, Solitons and Fractals 39 (2009) 1615-1626.

[25] T. Hayat, Z. Abbas, N. Alib, MHD flow and mass transfer of a upper-convected Maxwell fluid past a porous shrinking sheet with chemical reaction species, Physics Letters A 372 (2008) 4698-4704. 
[26] T. Hayat, T. Javed, M. Sajid, Analytic solution for MHD rotating flow of a second grade fluid over a shrinking surface, Physics Letters A 372 (2008) 3264-3273. MR2414279

[27] S. Nadeem, M. Awais, Thin film flow of an unsteady shrinking sheet through porous medium with variable viscosity, Physics Letters A 372 (2008) 4965-4972.

[28] N.F.M. Noor, S. Awang Kechil, I. Hashim, Simple non-perturbative solution for MHD viscous flow due to a shrinking sheet, Communications in Nonlinear Science and Numerical Simulation (2009), doi: $10.1016 /$ j.cnsns.2009.03.034.

[29] M. Sajid, T. Hayat, The application of homotopy analysis method for MHD viscous flow due to a shrinking sheet, Chaos, Solitons and Fractals 39 (2009) 1317-1323.

[30] C.Y.Wang, Stagnation flow towards a shrinking sheet, International Journal of Non-Linear Mechanics 43 (2008) 377-382.

[31] M. Miklavcic, C. Y. Wang, Viscous flow due to a shrinking sheet. Quart. Appl. Math. 64 (2006) 283-290. MR 2243864(2007c:76021)

[32] I. Muhaimin, R. Kandasamy, A. B. Khamis. Effects of heat and mass transfer on nonlinear MHD boundary layer flow over a shrinking sheet in the presence of suction. Appl. Math. Mech. -Engl. Ed. 29 (2008) 1309-1317.

[33] M. Rahimpour, S. R. Mohebpour, A. Kimiaeifar, G. H. Bagheri. On the analytical solution of axisymmetric stagnation flow towards a shrinking sheet, International Journal of Mechanics 2 (2008) $1-10$.

[34] J.A. Shercliff, in: A Textbook of Magnetohydrodynamics, Pergamon Press, London, 1965, pp. 45-50. MR0185961 (32:3421)

[35] S.J. Liao, A new branch of solutions of boundary-layer flows over a stretching flat plate, Int. J. Heat Mass Transfer 49 (2005) 2529-2539.

[36] S.J. Liao, A new branch of solution of boundary-layer flows over a permeable stretching plate, Int. J. Non-Linear Mech. 42 (2007) 819-830. MR2328735

[37] L.J. Crane, Flow past a stretching plate, Z. Angew. Math. Phys. 21 (1970) 645.

[38] M. S. Abel, M. M. Nandeppanavar, Heat transfer in MHD viscoelastic boundary layer flow over a stretching sheet with non-uniform heat source/sink, Communications in Nonlinear Science and Numerical Simulation 4 (2009) 2120-2131. MR2474469

[39] C.Y. Wang, Exact solutions of the steady state Navier-Stokes equations, Ann. Rev. Fluid Mech. 23 (1991) 159-177. MR1090331 (92a:76030)

[40] A. Chakrabarti, A.S. Gupta, Hydromagnetic flow and heat transfer over a stretching sheet, Quart. Appl. Math. 37 (1979) 73-78.

[41] K. Vajravelu, D. Rollins, Heat transfer in an electrically conducting fluid over a stretching sheet, Int. J. Non-Linear Mech. 27 (1992) 265-277.

[42] H.I. Andersson, An exact solution of the Navier-Stokes equations for magnetohydrodynamic flow, Acta Mech. 113 (1995) 241-244. MR:1361702 (96h:76082)

[43] I. Pop and T.Y. Na, A note on MHD flow over a stretching permeable surface, Mech. Res. Commun. 25 (1998) 263-269. MR1618160

[44] S.J. Liao, On the analytic solution of magnetohydrodynamic flows of non-Newtonian fluids over a stretching sheet, J. Fluid Mech. 488 (2003) 189-212. MR2019664 (2004j:76169) 